\title{
Caracterisation des Agrosystemes de la Commune de Gogounou au Nord-Est du Benin par Teledetection a Moyenne Resolution Spatiale
}

\author{
André Kindjinou \\ Département de Géographie et Aménagement du Territoire (DGAT), Benin \\ Hervé Koumassi \\ Cyr Gervais Etene \\ Ibourä̈ma Yabi \\ Laboratoire Pierre PAGNEY, Climat, Eau, Ecosystème et Développement, \\ Université d'Abomey-Calavi, Cotonou 1, Benin \\ Ismaïla Toko Imorou \\ Laboratoire de Cartographie (LaCarto), \\ Université d'Abomey-Calavi, Cotonou-Houéyiho, Benin
}

Doi:10.19044/esj.2021.v17n14p203

Submitted: 16 September 2020

Accepted: 23 March 2021

Published: 30 April 2021
Copyright 2021 Author(s)

Under Creative Commons BY-NC-ND

4.0 OPEN ACCESS

Cite As:

Kindjinou A., Koumassi H., Etene C.G., Yabi I. \& Imorou I.T. (2021). Caracterisation des Agrosystemes de la Commune de Gogounou au Nord-Est du Benin par Teledetection a Moyenne Resolution Spatiale. European Scientific Journal, ESJ, 17(14), 203.

https://doi.org/10.19044/esj.2021.v17n14p203

\section{Resume}

La télédétection se présente de plus en plus comme un outil précieux d'analyse des agrosystèmes en complément des approches traditionnellement utilisées. La présente étude vise à caractériser les agrosystèmes de la commune de Gogounou à partir des données de télédétection. La méthodologie développée a consisté à l'utilisation des séries temporelles d'images SPOT6 et SPOT7 (6 $\mathrm{m}$ de résolution spatiale) acquises sur la commune de Gogounou et des données secondaires issues de la base de données du projet OSFACO et des points GPS de 85 exploitations agricoles réparties dans la commune de Gogounou. La cartographie du domaine cultivé a été réalisée à partir d'indices spectraux dérivés des images SPOT. Trois types de systèmes agricoles ont été déterminés : les cultures vivrières (maïs, mil, sorgho) représentant $66 \%$ des zones agricoles, la culture de rente (coton) pour $13 \%$ du secteur et les cultures 
mixtes (mélange de sorgho, de mil et de coton) représentant $21 \%$. Les résultats de cette étude permettent de dégager de nouvelles pistes de couplage de télédétection et données de terrain en apportant une information continue dans le temps sur la caractérisation des agrosystèmes au Bénin.

Mots-clés : Gogounou, cartographie, agrosystème, indices de télédétection, domaine cultivé

\title{
Characterization of Agrosystems in the Municipality of Gogounou in Northeast Benin by Medium-Resolution Remote Sensing
}

\author{
André Kindjinou \\ Département de Géographie et Aménagement du Territoire (DGAT), Benin \\ Hervé Koumassi \\ Cyr Gervais Etene \\ Ibourä̈ma Yabi \\ Laboratoire Pierre PAGNEY, Climat, Eau, Ecosystème et Développement, \\ Université d'Abomey-Calavi, Cotonou 1, Benin \\ Ismaïla Toko Imorou \\ Laboratoire de Cartographie (LaCarto), \\ Université d'Abomey-Calavi, Cotonou-Houéyiho, Benin
}

\begin{abstract}
Remote sensing is increasingly presented as a valuable tool for analyzing agrosystems, in addition to the approaches traditionally used. The present study aims to characterize the agrosystems of the commune of Gogounou from remote sensing data. The methodology developed consisted in using the time series of SPOT6 and SPOT7 images (6 m spatial resolution) acquired in the town of Gogounou and secondary data made up of the OSFACO project database and GPS points of 85 farms spread throughout the area. Mapping of the cultivated area was carried out using spectral indices derived from SPOT images. Three types of agricultural systems were identified: food crops (maize, millet, sorghum) representing 66\% of agricultural areas, cash crops (cotton) for $13 \%$ of the sector and mixed crops (mixture of sorghum, millet and cotton) representing 21\%. The results of this study make it possible to identify new avenues for coupling remote sensing and field data by providing continuous information over time on the characterization of agrosystems in Benin.
\end{abstract}


Keywords: Gogounou, mapping, agrosystem, remote sensing indices, crop area

\section{Introduction}

L'existence de la vie humaine est tributaire aux ressources de la Terre. Parmi ces dernières, l'on note les ressources alimentaires qui proviennent des activités de production des hommes. On y distingue la foresterie, les activités de transformation alimentaire, la production animale et la production végétale (Kouhoundji et al., 2016). La satisfaction des besoins alimentaires constitue un défi pour l'humanité et se repose sur la production agricole. En Afrique Tropicale et particulièrement au Bénin, le système agricole est constitué d'une agriculture vivrière de subsistance essentiellement céréalière (Akponikpe et al., 2019), tributaire du climat et reposant sur des équilibres écologiques de plus en plus fragiles (Edea, 2018). Au Bénin, dans le but de satisfaire les besoins alimentaires de sa population, les gouvernants ont élaboré un Plan Stratégique de Développement du Secteur Agricole (PSDSA) à l'horizon 2025. Ce plan vise à assurer une intercommunalité plus bénéfique aux populations, grâce à l'amélioration de l'accès des acteurs des filières agricoles à des services de qualité revêtant un intérêt commun. Ainsi, la caractérisation des espaces agricoles est indispensable pour une mise en œuvre efficiente du PSDSA, car elle permet d'avoir dans le temps, la répartition géographique des zones agricoles d'un espace donné.

Le département de l'Alibori et plus précisément la commune de Gogounou ne fait pas exception à ces défis agricoles. En effet, dans la Commune de Gogounou, l'agriculture est extensive et se pratique sur des domaines cultivés fragmentés, avec des parcelles de petites tailles de 10 hectares soumises à la synchronisation des phénologies des agrosystèmes liées au régime des précipitations (Mama et al., 2013 ; Dedjan, 2010). Alors pour prévoir la production agricole, les caractéristiques des agrosystèmes doivent être renseignées par des données sur les surfaces cultivées (Begue et al., 2016). La télédétection se révèle particulièrement utile dans les domaines relatifs aux surfaces cultivées, aux rendements et à la production. La méthode classique d'estimation des surfaces cultivées est toutefois longue, coûteuse, fastidieuse et sujette aux erreurs humaines (Ray et Neetu. 2017). Elle est aussi extrêmement difficile à appliquer sur certains types de terrain, notamment les zones d'altitude basse. Face à ces difficultés, la télédétection par satellite offre une solution pour estimer les surfaces cultivées, soit directement ou indirectement en complément aux systèmes d'échantillonnage aréolaire.

Le recours à la télédétection aux fins d'identification et de classification des cultures, se justifie principalement par la différence d'apparence des cultures dans les données multibandes (Leroux, 2015). Cette variation s'explique par les caractéristiques structurelles, physiologiques et 
phénologiques des plantes ainsi que par la diversité des pratiques culturales (Vintrou et al., 2012). L'identification des cultures est rendue possible par l'association de ces données de télédétection et de la réalité de terrain collectée sur les sites échantillonnés. La présente étude vise à caractériser les agrosystèmes de la commune de Gogounou à partir d'images SPOT 6-7. Les images SPOT sont cohérentes en termes de période d'acquisition ensoleillée (en novembre et en décembre) et couvrent toute la commune de Gogounou. Ces images n'ont pratiquement pas de couvert nuageux.

\section{Caractéristiques générales du milieu}

La commune de Gogounou est située au sud du département de $l^{\prime}$ Alibori et s'étend sur les méridiens $2^{\circ} 24^{\prime}$ à $3^{\circ} 15^{\prime}$ Est les parallèles $10^{\circ} 32^{\prime}$ et $11^{\circ} 05^{\prime}$ Nord (figure 1 ).

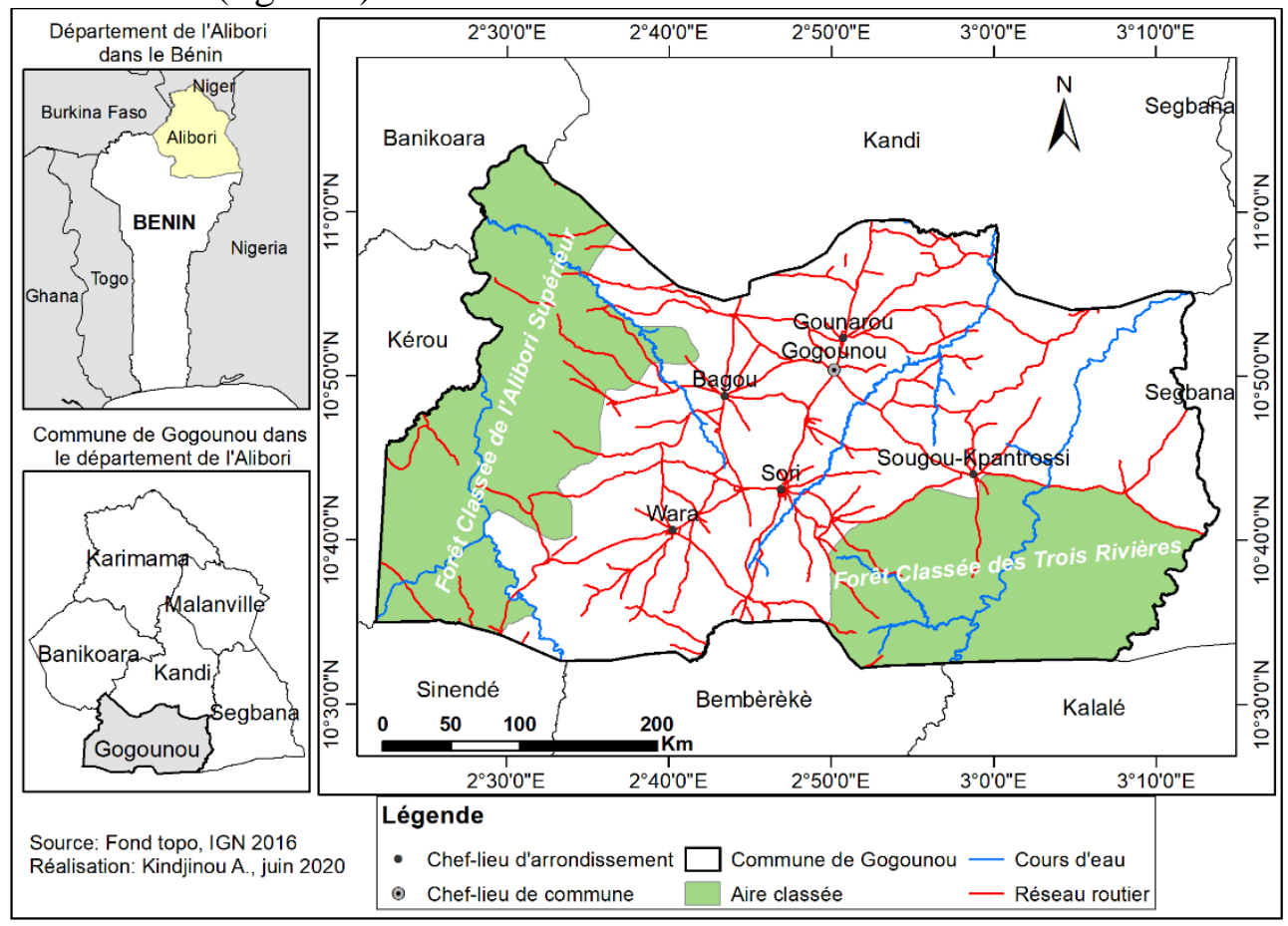

Figure 1 : Situation géographique et administrative de la Commune de Gogounou

Avec une population agricole estimée à 92085 actifs pour 10976 ménages agricoles, la commune de Gogounou couvre une superficie de 3803 $\mathrm{km}^{2}$, ce qui représente $18,66 \%$ de l'ensemble du département de l'Alibori (Adapté de IGN, 2016 ; MAEP, 2017).

Elle est limitée au nord par les communes de Banikoara et de Kandi, au sud par les communes de Sinendé et de Bembéréké, à l'est par les communes de Ségbana et de Kalalé puis à l'ouest par la commune de Kérou. 
Elle est située dans le domaine climatique soudano-guinéen marqué par une saison pluvieuse de mai à octobre (en moyenne $105 \mathrm{~mm}$ en mai à $270 \mathrm{~mm}$ de pluie en septembre avec une température moyenne de $28^{\circ} \mathrm{C}$ ), et une saison sèche de novembre à avril. Les sols sont majoritairement ferrugineux (96 \%) généralement aptes pour la culture du coton, du maïs, du sorgho, du riz et du maraîchage (adapté de Azontondé et al., 2009). Le réseau hydrographique du secteur d'étude est essentiellement composé du fleuve Ouémé et des cours d'eau. En saison sèche, il existe des mares en chapelet dans les lits mineurs des grands cours d'eau. Par contre, l'assèchement de plusieurs cours d'eau secondaires durant cette période se justifie par la faiblesse du surplus hydrologique qui est en moyenne de 105,6 mm. Les aires classées occupent $41 \%\left(1557 \mathrm{Km}^{2}\right)$ de la superficie de la commune et la présente recherche exclue cette partie. L'agriculture et l'élevage sont les principales activités de la population dans la commune.

\section{Données et méthodes}

\subsection{Données}

Les données utilisées sont de natures quantitative et qualitative et sont issues de plusieurs sources. Les données qualitatives que sont, les types de cultures, l'association de cultures, les superficies emblavées, les pratiques agricoles et la durée du cycle des cultures sont issues des entretiens auprès de 50 exploitants agricoles produisant principalement le coton, le maïs, le mil, le sorgho et le riz. Quant aux données quantitatives elles sont composées de trois images SPOT 6-7 à $6 \mathrm{~m}$ de résolution, des données vectorielles d'occupation du sol issues de la base de données de la composante forêt galerie du projet OSFACO (Observation Spatiale des Forêts d'Afrique Centrale et de l'Ouest) 2016 et 85 points de vérité terrain représentant différents agrosystèmes dans 13 villages (figure 2 ). 


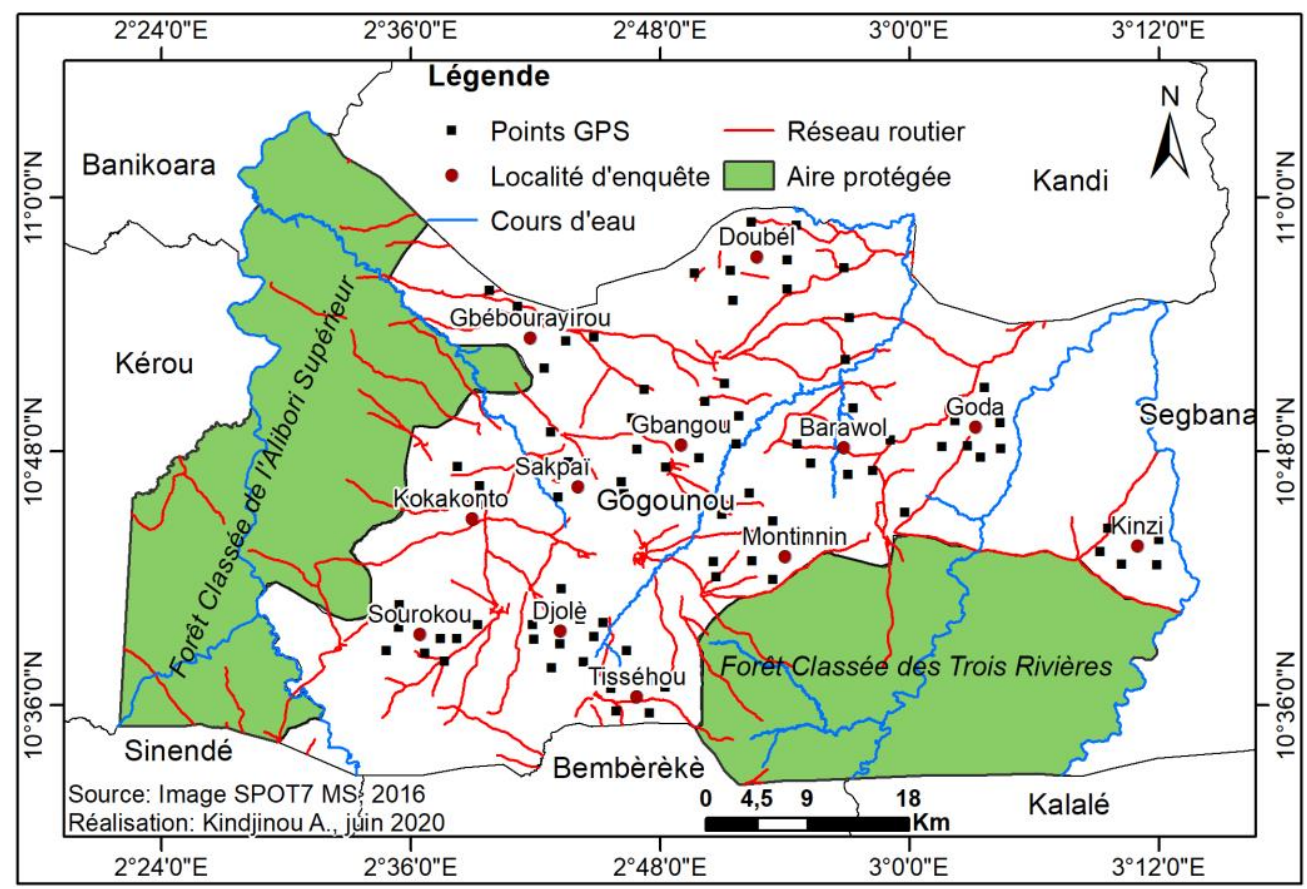

Figure 2 : Répartition géographique des points GPS et des localités d'enquête

Le tableau I présente les caractéristiques des images satellitaires utilisées.

Tableau I : Caractéristiques des images SPOT utilisées

\begin{tabular}{cccc}
\hline Image & Synthèses temporelles & Résolution spatiale & Date \\
\hline SPOT 6 MS & Annuel & $6 \mathrm{~m}$ & Décembre 2015 \\
\hline SPOT 7 MS & Annuel & $6 \mathrm{~m}$ & Novembre 2016 \\
\hline SPOT 7 MS & Annuel & $6 \mathrm{~m}$ & Décembre 2016 \\
\hline
\end{tabular}

Source : OSFACO, Images Pivot (2015-2016)

\subsection{Méthodes de traitement des données}

Pour caractériser les agrosystèmes de la commune de Gogounou, deux méthodes sont utilisées. La première approche est basée sur l'utilisation d'indice spectral contenu dans l'image SPOT 6-7 pour cartographier le domaine cultivé et le domaine non cultivé dans la commune de Gogounou. Cette méthode comprend deux étapes : la stratification du territoire et la classification culture et non culture. L'indice spectral NDVI (Normalized Difference Vegetation Index) de trois images SPOT7 des mois de novembre et décembre 2016 à $6 \mathrm{~m}$ de résolution est d'abord utilisé pour stratifier le territoire en zones homogènes. Cette stratification est faite avec le logiciel QGIS 2.18.4 en procédant à une segmentation et à une classification supervisée des segments. 
Le NDVI et l'EVI sont calculés de la façon suivante :

$$
N D V I=\frac{P I R-R}{P I R+R} \in[-1,1]
$$

Pour le calcul des valeurs de NDVI, le fichier VRT regroupant les 3 bandes du visible (x1, x2 et x3) et la bande de l'infrarouge (x4) est utilisé avec la fonction construire un raster visuel. Par la suite, dans la calculatrice raster de QGIS, les opérations de calcul sont effectuées avec enregistrement de nouvelles images.

Les résultats de classification sont évalués avec les mesures de précision pour l'utilisateur et pour le réalisateur en utilisant la base de données (BD) du projet OSFACO et les données de terrain.

La seconde approche repose sur la superposition pixel par pixel des indices NDVI et de la base de données vectorielles et celles des points de vérité terrain. Elle utilise l'Analyse en Composante Principale (ACP) des données issues des entretiens avec les paysans pour définir trois types de systèmes agricoles. Chacun des 85 points a été assigné à une classe $\mathrm{U}_{1}, \mathrm{U}_{2}$ et $\mathrm{U}_{3}$ en considérant les cultures majoritairement cultivées dans ces agrosystèmes :

- la classe $\mathrm{U}_{1}$ regroupe les champs de coton ;

- la classe $\mathrm{U}_{2}$ regroupe les champs de sorgho, de maïs et de mil avec le maïs comme culture majoritairement cultivée ;

- la classe $\mathrm{U}_{3}$ qui est une classe mixte est composée à la fois des cultures vivrières (mil, sorgho, maïs) et le coton.

\section{Résultats et discussions}

\subsection{Zonage du domaine agricole de la commune de Gogounou}

La méthode de cartographie a permis de montrer l'importance des SPOT 6-7 dans l'identification du domaine cultivé. Dans l'ensemble, les résultats sont satisfaisants (figure 3). 


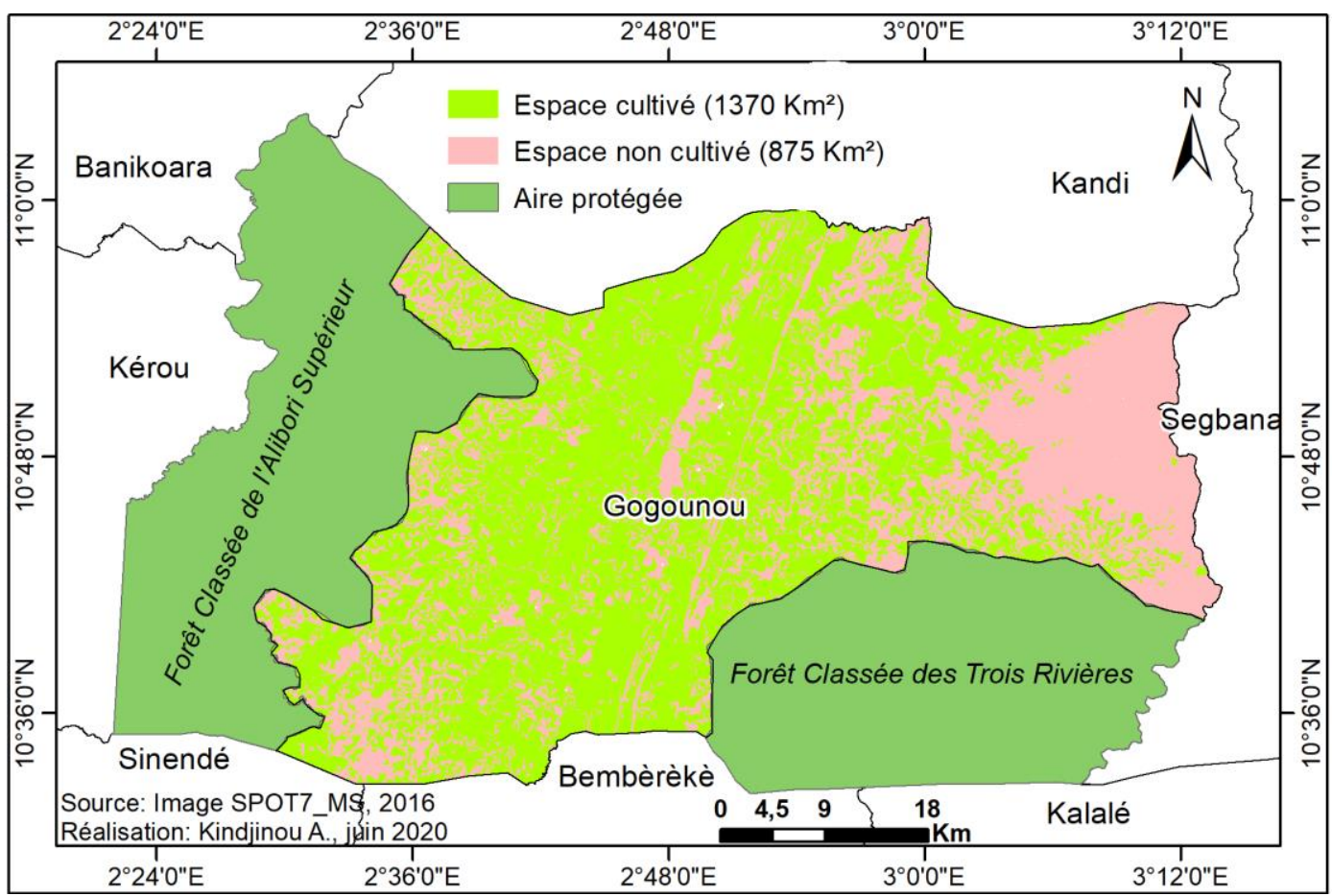

Figure 3: Stratification de la commune de Gogounou en deux zones homogènes selon le NDVI $875 \mathrm{~km}^{2}$.

Les espaces cultivés occupent $1370 \mathrm{~km}^{2}$ et les espaces non cultivés font

Les résultats de la classification ne présentent pas le même niveau de satisfaction selon le lieu dans la commune.

La carte du domaine cultivé issue de la classification a été validée avec les données d'OSFACO en discriminant les espaces cultivés des espaces non cultivés dans la commune de Gogounou. Cette comparaison s'est faite en utilisant la matrice de confusion (tableau II).

Tableau II : Matrice de confusion entre NDVI et la BD OSFACO

\begin{tabular}{|c|c|c|c|}
\hline & & NDVI & BD OSFACO \\
\hline \multirow{2}{*}{ Espace cultivé } & Erreur d'omission & $36,1 \%$ & $67,3 \%$ \\
\hline & Erreur de commission & $38,5 \%$ & $28,4 \%$ \\
\hline \multirow{2}{*}{ Espace non cultivé } & Erreur d'omission & $27,4 \%$ & $19,7 \%$ \\
\hline & Erreur de commission & $24,6 \%$ & $42,6 \%$ \\
\hline \multicolumn{2}{|c|}{ Pourcentage de Pixels correctement classés } & $69,7 \%$ & $52,5 \%$ \\
\hline \multicolumn{2}{|c|}{ F-Score } & 0,619 & 0,468 \\
\hline
\end{tabular}

Source : Résultats de traitement d'images, décembre 2019 
La matrice de confusion montre que pour la classe espaces cultivés, NDVI de SPOT minimise les erreurs d'omission (36,1\% contre 67,3\%), alors que la BD OSFAC minimise celles de la commission $(28,4 \%$ contre $38,5 \%)$. La proportion de pixels correctement classées est supérieure pour les données NDVI (69,7\% contre 52,5\%). Les erreurs de classification de la classe espace non cultivé sont comprises entre 24 et $27,4 \%$ pour l'approche NDVI et entre 19 et $42,6 \%$ pour l'approche BD OSFACO.

Une seconde phase de validation est basée sur les points de vérité terrain. Les résultats de la classification ont été comparés avec les 85 points GPS en utilisant les mesures de précision pour l'utilisateur et pour le réalisateur (tableau III).

Tableau III : Validation des résultats de classification avec les données de terrain

\begin{tabular}{cccc}
\hline Approche & Précision utilisateur & Précision réalisateur & F-Score \\
\hline NDVI & 0,695 & 0,689 & 0,689 \\
Points GPS & 0,689 & 0,787 & 0,702 \\
\hline
\end{tabular}

L'analyse du tableau III montre que la valeur de F-Score de NDVI $(0,689)$ est légèrement inférieure à celle de la F-Score des points GPS . Cependant, l'analyse des résultats de Précision pour l'utilisateur et de Précision pour le réalisateur permet d'observer que l'utilisation de NDVI offre des résultats plus stables, avec une Précision utilisateur presque égale à la Précision réalisateur. Ainsi, le domaine cultivé a été bien déterminé à l'aide de NDVI des images SPOT7.

\subsection{Principaux systèmes agricoles de la commune de Gogounou}

L'ACP a été réalisée sur les variables les plus discriminantes. Les deux premiers axes montrent, la superficie emblavée, les types de culture et la pratique agricole. Une classification est issue de cette technique en distinguant deux classes. Ainsi, une première classe (U1) regroupant les points où se cultive le coton à $95 \%$ a été constituée. Elle est suivie d'une deuxième classe (U2), représentant les points du domaine cultivé est composé de maïs (32\%), de sorgho $(29 \%)$ et de mil (25\%). Enfin, la classe U3, celle des cultures mixtes, regroupe l'ensemble des points où se trouvent les champs de coton (20 $\%$ ), de sorgho ( $23 \%$ ), de maïs ( $27 \%$ ) et de coton (30\%). Chacun des 85 points de terrain, selon le critère de culture majoritaire, a été assigné à une des trois classes.

\subsection{Cartographie des agrosystèmes de la commune de Gogounou}

La cartographie des agrosystèmes résultante de la méthode utilisée distingue les principales zones d'agriculture (Figure 4). 


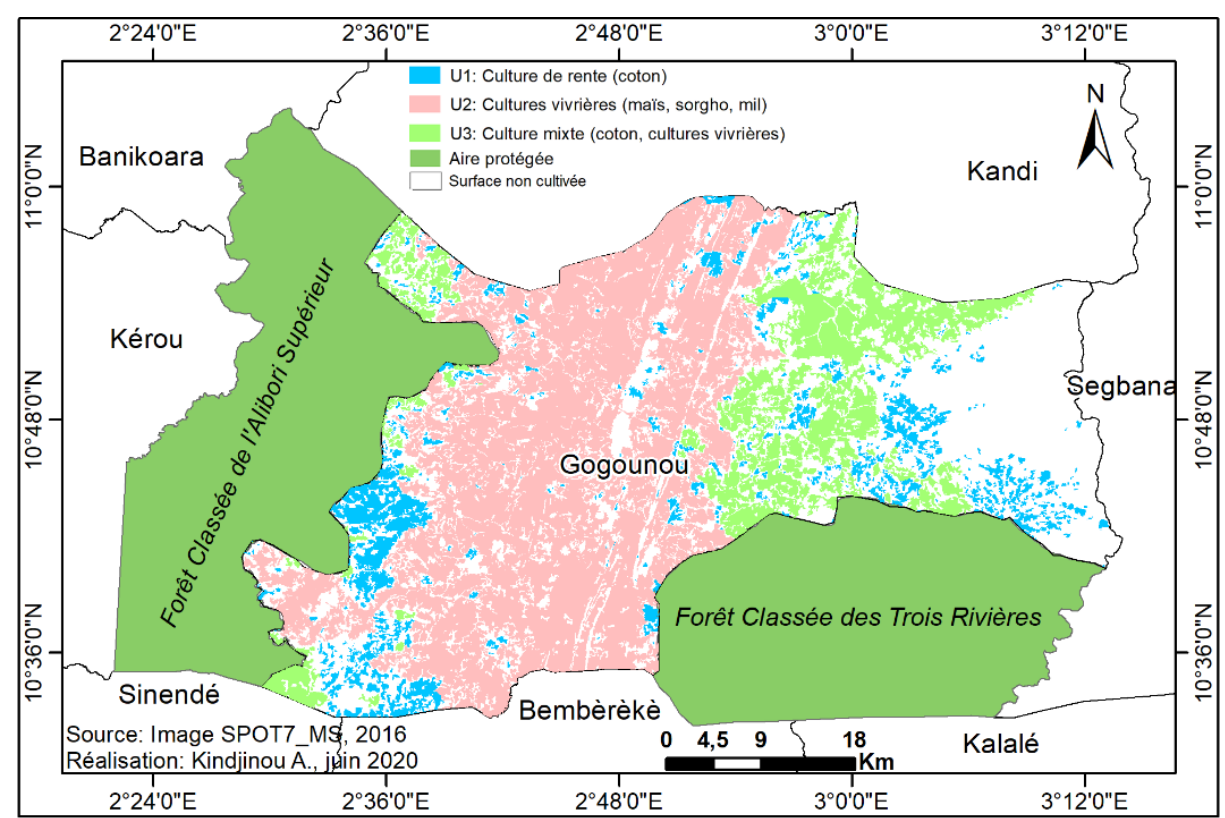

Figure 4 : Cartographie des agrosystèmes de la commune de Gogounou

Trois principales zones agricoles se distinguent :

- le centre de la commune, où la classe U2 prédomine (66 \%). En effet, les cultures vivrières notamment le maïs, le sorgho, le riz et le mil y sont cultivées. Dans cette classe, se trouvent des champs de maraîchage avec la présence d'espèces pérennes (planche 1c);

- la classe U1 est répartie sur tout le secteur d'étude et occupe $13 \%$ de la superficie soit $172 \mathrm{~km}^{2}$. Dans cette classe se pratique la culture du coton (planche 1d);

- le reste du secteur d'étude est occupé par la classe U3 qui représente $21 \%$ de la superficie totale. Les systèmes agricoles sont centrés sur la production de coton (classe U1) associée à des cultures vivrières (classe U2).

La planche 1 est une illustration non exhaustive de différents paysages agricoles rencontrés lors des missions de contrôle terrain. 

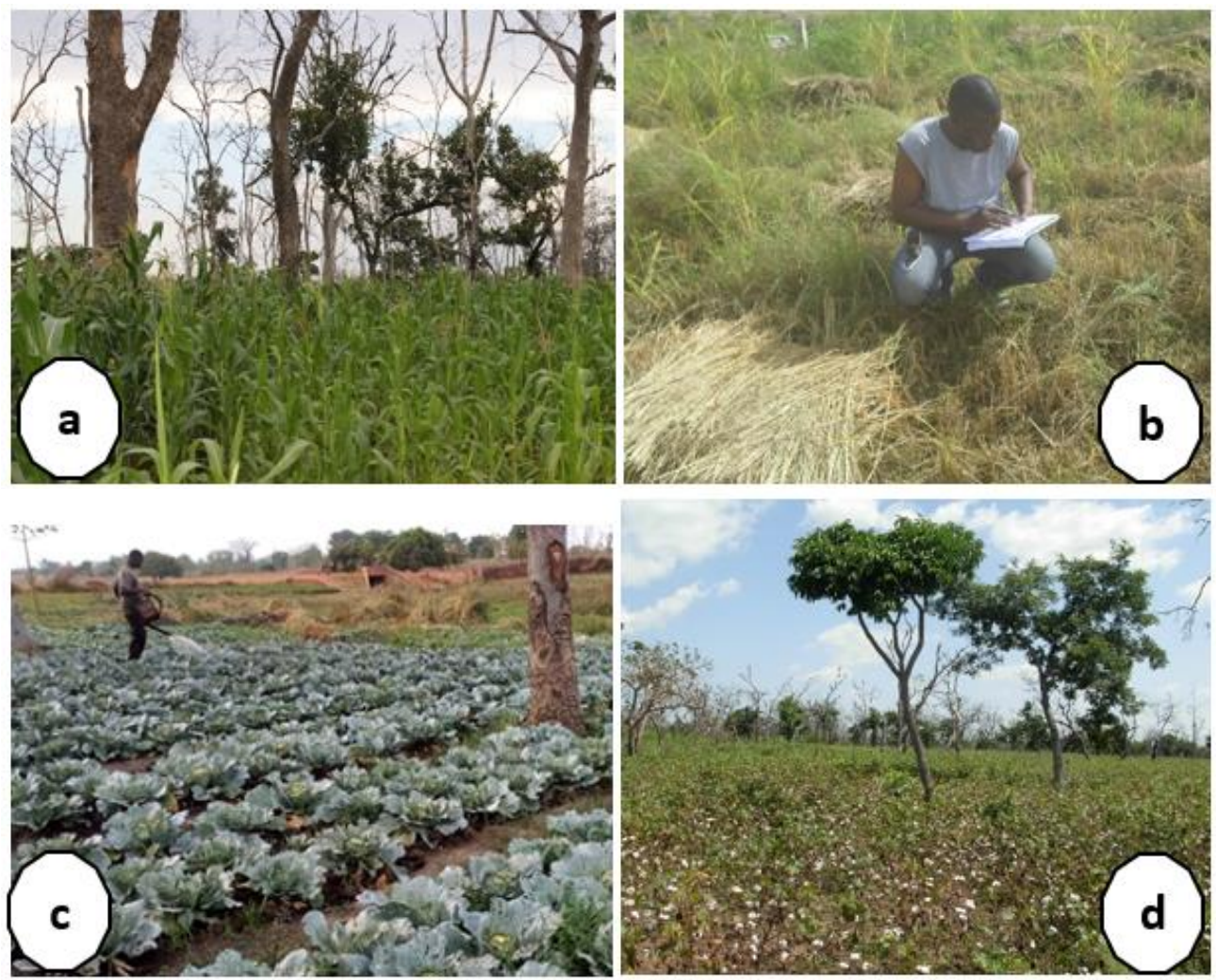

Planche 1: Illustration de quelques paysages agricoles rencontrés lors des missions de terrain effectuées sur les sites de recherche dans la commune de Gogounou

Prise de vue : Kindjinou, octobre 2019

La planche (1a) présente un champ de maïs (Zea mays) dans le village Garagoro. Dans ce champ se trouvent douze pieds de Karité (Vitellaria paradoxa), une espèce pérenne dont le beurre obtenu de sa transformation est consommé localement ou exporté pour les cosmétiques. La planche (1b), quant à elle, montre une prise de coordonnées GPS dans un champ de riz (Oryza sativa) en début de récolte dans le village Badou. La planche (1c), présente une image montrant un paysan entrain d'arroser un champ de choux (Brassica oleracea $L$, ) à Ouèrè-Sonkérou. La dernière planche (1d) présente la culture du cotonnier après semis précoce (1 ${ }^{\text {ère }}$ décade de mai) à SomboaGasso. La méthode développée a permis de cartographier les principaux agrosystèmes de la commune de Gogounou à partir du NDVI de SPOT 6-7 à $6 \mathrm{~m}$ de résolution spatiale et des données secondaires.

\section{Discussion}

La cartographie des agrosystèmes de la commune de Gogounou a nécessité dans un premier temps la classification des images SPOT7 pour le calcul du NDVI. Dans un second temps, les données de classification sont 
comparées à la base de données OSFACO et les points GPS de terrain. Trois types d'agrosystème ont été identifiés. Il s'agit des agrosystèmes de coton, des agrosystèmes de cultures vivrières (maïs, sorgho, riz et mil) et des agrosystèmes mixtes (coton et cultures vivrières). Les résultats de validation de la cartographie sont de bonne précision (61\%). Cette précision confirme l'hypothèse de départ qui postule qu'un agrosystème se caractérise par des indices spectraux et qu'il est possible de la distinguer à partir des images SPOT 6-7. Ces résultats peuvent être expliqués d'une part, par la fragmentation et l'hétérogénéité des agrosystèmes pour être détectés par SPOT 6-7 ou séparés de la végétation naturelle. Ces deux facteurs ont été confirmés par Edea (2018) et Leroux et al., (2014). D'autre part, les résultats montrent des erreurs de photo-interprétation à partir des images SPOT 6-7. L'approche utilisant la BD OSFACO a permis de mettre en exergue la corrélation entre occupation du sol et indice de télédétection. En effet, dans les travaux de Ray et Neetu, (2017), la texture des images s'est révélée être une dimension plus importante que l'indice spectral (NDVI) pour la distinction des espaces cultivés des espaces non cultivés. Cependant, de nombreuses difficultés continuent de limiter le recours aux données de télédétection dans les opérations d'évaluation des surfaces cultivées, telles que :

- la production agricole sur les parcelles de petites tailles qui nécessite l'utilisation des données de télédétection à haute résolution pour l'identification des cultures ;

- la persistance de la couverture nuageuse pendant la saison des pluies. Les nuages limitent fortement l'intérêt de l'imagerie optique pour les applications agricoles (Eberhardt, 2016) ;

- la diversité des pratiques culturales et agronomiques ;

- les systèmes de cultures mixtes et associées ;

- les petites surfaces incluant une grande variété de cultures, particulièrement dans le cas du maraîchage.

\section{Conclusion}

La méthode utilisée et les missions de terrain dans la commune de Gogounou ont permis de caractériser les agrosystèmes. De l'analyse des résultats, il ressort que les agrosystèmes de la commune de Gogounou sont caractérisés par la culture de maïs, du sorgho, du riz et du mil, le coton et les cultures mixtes. La culture vivrière est la principale activité agricole de la population. La carte des principaux agrosystèmes (cultures de rente, cultures vivrières et cultures mixtes) produite dans cette recherche est importante car elle est une source originale et indépendante d'information pour la commune de Gogounou. Etant donné que les facteurs locaux comme le climat, le sol, le relief, la disponibilité en eau, l'accès aux marchés et aux engrais, influencent les agrosystèmes, la cartographie de ces systèmes peut aider à déterminer les 
zones et les populations vulnérables aux risques d'inondation et de sécurité alimentaire. Il est alors essentiel de localiser ces systèmes afin de procéder à une analyse précise de l'état de la sécurité alimentaire face aux besoins de la population.

\section{References :}

1. Akponikpe P.B.I., Tovihoudji P., Lokonon B., Kpadonou E., Amegnaglo J., Segnon A. C., Yegbemey R., Hounsou M., Wabi M., Totin E., Fandohan-Bonou A., Dossa E., Ahoyo N., Laourou D., Aho N., 2019: Etude de Vulnérabilité aux changements climatiques du Secteur Agriculture au Bénin. "Projet d'Appui Scientifique aux processus de Plans Nationaux d'Adaptation dans les pays francophones les moins avancés d'Afrique subsaharienne”, Climate Analytics gGmbH, Berlin. $97 \mathrm{p}$

2. Azontondé H. A., Igué A. M. et Dagbénonbakin G., 2009 : Carte de fertilité des sols du Bénin par zone agro-écologique du Bénin. Rapport d'étude. LSSEE/CRA-AGONKANMEY/INRAB, AFRIQUEETUDES, 121p.

3. Begue A.., Leroux L., Lo Seen D., T. Tonneau J-P et Morant P., 2016 : Observation spatiale pour l'agriculture en Afrique : potentiels et défis, Paris cedex. Note Technique, 12, 185.

4. Dedjan Y.S., 2010: Changements climatiques et évolution des périodes de semis des principales cultures dans l'Alibori : cas des communes de Malanville et de Banikoara. Mémoire Economie et Sociologie Rurales : Université de Parakou, 122 p.

5. Eberhardt, I.D.R., 2016: Cloud Cover Assessment for Operational Crop Monitoring Systems in Tropical Areas ». Remote Sensing, vol. 8, n o 3, p. 219.

6. Edea O. E, 2018: Cartographie des systèmes agricoles par télédétection et prédiction des rendements dans les hydro-écorégions du bassin versant de l'Ouémé Supérieur au Bénin. Thèse de Doctorat Unique. /EDP/FASHS/UAC, 264 p.

February 2016, p.42-48.

7. Gallego, J. , 2017: La télédétection au service de la conception de bases de sondage ». In : J. Delincé (dir.), Manuel sur l'application de la télédétection aux statistiques agricoles (chapitre 3). Manuel de la Stratégie mondiale pour l'amélioration des statistiques agricoles et rurales (GSARS) : Rome.

8. Hoffman A. L., Kemanian A. R. et Forest C. E., 2017: Analysis of climate signals in the crop yield record of sub-Saharan Africa. Global Change Biology, 24(1): pp 143-157. 
9. IGN France (Institut Géographique National), 2016 : archives images SPOT, projet OSFACO.

10. INSAE, 2015 : RGPH4 : que retenir des effectifs de population en 2013 ? Rapport, Direction des Etudes Démographiques, Cotonou, Bénin, 35p.

11. Kouhoundji N., Sintondji L. O., Vissin E. W., Agbahungba G. A., 2016: Geostatistical analysis

12. Leroux L., 2015 : Suivi et Caractérisation des Dynamiques de la Production Agricole en Afrique de l'Ouest par Télédétection à Moyenne Résolution Spatiale. Thèse de doctorat Paris Tech, Institut des Sciences et Industries du Vivant et de l'Environnement, 285 p.

13. Leroux L., Jovilot A., Bégué A., Lo Seen D. et Zoungrana B., 2014 : How Reliable is the MODIS Land Cover Product for Crop Mapping Sub-Saharan Agricultural Landscapes? Remote Sens. 2014, 6, 85418564.

14. MAEP (Ministère de l'Agriculture, de l'Elevage et de la Pêche), 2017 : Plan Stratégique de Développement du Secteur Agricole (PSDSA) 2025 et Plan National d'Investissements Agricoles et de Sécurité Alimentaire et Nutritionnelle (PNIASAN) 2017-2021. Cotonou, Bénin, $139 \mathrm{p}$.

15. Mama A., Sinsin B., De Cannière C. \& Bogaert J., 2013 : Anthropisation et dynamique des paysages en zone soudanienne au Nord du Bénin. Tropicultura, 31: 82-92.

of rainfall variability on the plateau of Allada in South Benin. In Int. Journal of Engineering

16. Ray S. et Neetu, 2017 : Estimation des surfaces cultivées à partir des données de télédétection ». In : J. Delincé (dir.), Manuel sur l'application de la télédétection aux statistiques agricoles (chapitre 5). Manuel de la Stratégie mondiale pour l'amélioration des statistiques agricoles et rurales (GSARS) : Rome, pp 133-163.

17. Research and Applications www.ijera.com ISSN: 2248-9622, Vol. 6, Issue 2, (Part - 4)

18. Stratégie mondiale pour l'amélioration des statistiques agricoles et rurales (GSARS), 2017 : Manuel sur l'application de la télédétection aux statistiques agricoles. Manuel de la Stratégie mondiale : Rome, $288 \mathrm{p}$.

19. Vintrou, E., Soumaré, M., Bernard, S., Bégué, A., Baron, C., Lo Seen, D., 2012 : Mapping Fragmented Agricultural Systems in the SudanoSahelian region using Random Forest and Metrics of Coarse Resolution MODIS Imagery. (Special Issue) Photogrammetric Engineering and Remote Sensing, $30 \mathrm{p}$. 